DOI: 10.12957/demetra.2015.18381

\title{
Perfil nutricional e consumo de alimentos inflamatórios e anti-inflamatórios de pacientes atendidos no ambulatório de psoríase de uma unidade de saúde-escola de Itajaí, SC
}

\author{
Nutritional profile and consumption of inflammatory and anti-inflammatory food of patients \\ treated in an outpatient clinic of psoriasis health-school unit in Itajaí, SC, Brazil
}

\author{
Katarine de Faria Odierno \\ Bruna Elisa Harmel de Borba Coelho \\ Cristina Henschel de Matos' \\ 1 Universidade do Vale do Itajaí, Centro de Ciências \\ da Saúde, Curso de Nutrição. Itajái-SC, Brasil. \\ Projeto financiado pela Bolsa de Pesquisa do \\ Artigo 170 da Constituição Estadual de Santa \\ Catarina de acordo com as Leis Complementares \\ $n^{0} 281 / 2005, n^{0} 296 / 2005$ e n ${ }^{0} 420 / 2008$. \\ Correspondência / Correspondence \\ Cristina Henschel de Matos \\ Clínica de Nutrição/ Curso de Nutrição, Centro de \\ Ciências da Saúde \\ Rua Uruguai 458, Centro, Itajaí-SC, Brasil CEP: \\ 88302-202 \\ E-mail:matoscris@gmail.com
}

\section{Resumo}

A psoríase é uma doença inflamatória de pele, mediada por células $\mathrm{T}$, hereditária, que pode sofrer influências ambientais. A psoríase e obesidade possuem uma fisiopatologia subjacente comum, sugerindo que obesidade segue psoríase, não a precede; além disto, há evidências de que determinados nutrientes, com função anti-inflamatória, podem auxiliar no tratamento da psoríase. O presente estudo objetivou avaliar o estado nutricional e o consumo de alimentos inflamatórios e anti-inflamatórios de pacientes com diagnóstico de psoríase atendidos em uma Unidade de Saúde-Escola de Itajaí-SC, de maio a agosto de 2014. Os pacientes atendidos foram avaliados por meio de um questionário semiestruturado, com dados de identificação, socioeconômicos, hábitos de vida e frequência do consumo de alimentos (inflamatório e anti-inflamatórios), sendo foi realizada avaliação do estado nutricional. A análise estatística foi efetuada por meio do programa STATA 13.0 e a associação entre as variáveis categóricas foi avaliada pelo teste do qui-quadrado de Pearson ou teste exato de Fisher. O excesso de peso prevaleceu em $61 \%$ dos pacientes, sendo $22 \%$ com sobrepeso e $39 \%$ com obesidade. Os resultados demonstram que o alho (97\%) e o azeite de oliva (53\%) foram os alimentos anti-inflamatórios consumidos com maior frequência, enquanto que os demais alimentos apresentaram baixo consumo. Quando questionados sobre o consumo de alimentos inflamatórios, o mais citado foi carne de gado, com 58\%, seguida pelo café (45\%); os demais alimentos não demonstraram relevância no consumo. Diante da magnitude do tema estudado, torna-se essencial o desenvolvimento de pesquisas e estudos 
relacionados ao estado nutricional de pacientes diagnosticados com psoríase e determinar o consumo de alimentos que possam auxiliar na melhora do tratamento desta patologia.

Palavras-chave: Psoríase. Alimentos. Estado Nutricional. Inflamação.

\section{Abstract}

Psoriasis is an inflammatory skin disease mediated by $\mathrm{T}$ cells, hereditary, that may suffer environmental influences. Psoriasis and obesity have a common underlying pathophysiology, suggesting that obesity follows psoriasis, not the contrary. Furthermore, there is evidence that certain nutrients with antiinflammatory function can assist in the treatment of psoriasis. This study aimed to evaluate the nutritional status and food intake of inflammatory and anti-inflammatory patients with psoriasis seen at a Health-School Unit of Itajaí-SC, from May to August 2014. The patients enrolled were evaluated by through a semi-structured questionnaire with identification, socioeconomic, lifestyle habits and frequency of food consumption (inflammatory and anti-inflammatory) data, and was carried out assessment of nutritional status. Statistical analysis was performed using the STATA 13.0 and the association between categorical variables was evaluated by chi-square test of Pearson or Fisher's exact test. Overweight prevailed in $61 \%$ of patients, $22 \%$ were overweight and $39 \%$ were obese. The results demonstrate that garlic (97\%) and olive oil (53\%) were anti-inflammatory food consumed more frequently, while others had lower food consumption. When asked about the use of inflammatory foods, the most cited was beef, with $58 \%$, followed by coffee (45\%); other foods showed no relevant consumption. Considering the magnitude of the studied subject, it is essential to the development of research and studies related to the nutritional status of patients diagnosed with psoriasis and determine the consumption of foods that can help improve the treatment of this pathology.

Key words: Psoriasis. Food. Nutritional Status. Inflammation. 


\section{Introdução}

A psoríase é uma doença inflamatória crônica, autoimune, de etiologia multifatorial e tem forte base genética; afeta a pele, as unhas, as mucosas e as articulações. ${ }^{1}$ Para Silva \& Silva, ${ }^{2}$ ela é caracterizada por uma placa eritemato-escamosa, saliente em relação à superfície da pele. Suas escamas estão superpostas como lâminas de um fragmento de mica; apresentam cor avermelhada e podem ser destacadas com facilidade, mediante a raspagem da lesão. Segundo Miot, ${ }^{1}$ a psoríase acomete em média 0,2\% a 4,8\% da população mundial. No Brasil, estima-se que cerca de 2,5\% de seus habitantes sejam acometidos pela doença.,

A inadequação do padrão alimentar, a inatividade física e os fatores emocionais podem desempenhar papel de gatilho para o desenvolvimento da psoríase e doenças crônicas não transmissíveis (DCNTs) associadas, bem como incrementar o processo inflamatório. Assim, a nutrição pode influenciar a psoríase de duas maneiras diferentes: como causa das desordens metabólicas ou como tratamento e prevenção.

O desconhecimento da importância dos alimentos, de realizar refeições regulares, a grande variedade de alimentos prontos com excesso de calorias e carboidratos e a falta de determinação para conciliar horários de trabalho e alimentação são fatores que, nesses pacientes, contribuem para a obesidade, deficiência nutricional e piora da psoríase. ${ }^{5}$ Segundo Sterry, Strober \& Menter, ${ }^{6}$ psoríase e obesidade possuem uma fisiopatologia subjacente comum, sugerindo que obesidade segue psoríase, não a precede, implicando que a inflamação psoriásica contribui para o estado obeso e ambas compartilham citocinas que competem para o padrão da síndrome metabólica.

A dieta é fator importante na patogênese da psoríase e há evidências na literatura que certos nutrientes regem grande influência nessa patogenia, como a exemplo do ácido graxo ômega 3, potente agente anti-inflamatório. Este pode ser empregado com sucesso no tratamento de doenças inflamatórias, mostrando melhorias no quadro clínico e significativa contribuição na diminuição das lesões dos pacientes. ${ }^{7,8}$

Em vista disso, a presente pesquisa visou avaliar o estado nutricional e o consumo de alimentos inflamatórios e anti-inflamatórios de pacientes com diagnóstico de psoríase atendidos em uma Unidade de Saúde-Escola de Itajaí-SC. 


\section{Metodologia}

Trata-se de estudo transversal e descritivo, constituído por indivíduos assistidos por um ambulatório de psoríase de uma Unidade de Saúde Escola no município de Itajaí-SC no período de maio a agosto de 2014. O projeto foi aprovado pelo Comitê de Ética em Pesquisa da UNIVALI, sob o parecer n⿳0 609.707 .

Para participar da pesquisa, os indivíduos deveriam ter 20 anos ou mais, ser atendidos pelo ambulatório de psoríase com diagnóstico confirmado, aceitar participar da pesquisa e assinar o Termo de Consentimento Livre e Esclarecido (TCLE). Não poderiam participar indivíduos nas seguintes condições: condição psiquiátrica ou neurocognitiva que impeça a obtenção de dados clínicos fidedignos (definida pelo julgamento clínico dos investigados); gravidez ou lactação; apresentar dificuldade de alimentação via oral, ser cadeirante; ter realizado gastroplastia ou transplante de órgão e apresentar diagnóstico de insuficiência renal com indicação de diálise.

A coleta de dados foi realizada pelos pesquisadores e teve abrangência nos aspectos relacionados a identificação, características socioeconômicas, hábitos de vida e saúde. Para a avaliação desses itens, foi aplicado um questionário semiestruturado na forma de entrevista, contendo questões referentes a identificação, dados socioeconômicos (renda e escolaridade), conforme modelo preliminar da Associação Brasileira de Empresas de Pesquisa (ABEP) ${ }^{9}$ e dados relacionados aos hábitos de vida (prática de atividade física e tabagismo).

O estado nutricional foi avaliado por meio de verificação do peso corporal $(\mathrm{kg})$ utilizando balança digital, com capacidade para $150 \mathrm{Kg}$, e os indivíduos foram avaliados com roupas leves e sem sapatos, em pé, no centro da plataforma da balança. A estatura $(\mathrm{cm})$ foi mensurada com um estadiômetro fixado à parede com escala em milímetros $(\mathrm{mm})$, estando o indivíduo em posição ortostática. Para o diagnóstico nutricional, foram utilizados o índice de massa corporal (IMC) e a classificação de acordo com os critérios propostos pela pelo Ministério da Saúde. ${ }^{10}$ Para fins estatísticos, os diagnósticos foram agrupados em baixo-peso e eutrofia, sobrepeso e obesidade. Por fim, foi avaliado o consumo de alimentos inflamatórios e anti-inflamatórios, por meio de um questionário de consumo alimentar de natureza semiquantitativa, contendo os principais alimentos presentes na literatura. ${ }^{4,5}$ Estes foram divididos em três categorias de consumo $\geq 3 \mathrm{x}$ semana, $<3 \mathrm{x}$ semana, não consome, tabulados e apresentados em percentual de consumo.

Os resultados da pesquisa foram tabulados com o auxílio do programa Microsoft Excel ${ }^{\circledR}$ e a análise estatística foi realizada pelo programa STATA $13.0^{\circledR}$. As variáveis quantitativas foram calculadas em médias e desvios-padrão. As variáveis categóricas foram descritas por meio de suas frequências absoluta e relativa. A associação entre as variáveis categóricas foi testada por meio do teste do qui-quadrado de Pearson, ou do teste exato de Fisher. Foram consideradas significativas as diferenças quando valor de $\mathrm{p} \leq 0,05$. 


\section{Resultados e Discussões}

Para realização do estudo, foram abordados 41 dos 55 pacientes cadastrados no ambulatório, mas apenas 36 aceitaram participar da pesquisa, dos quais $53 \%(n=19)$ eram do sexo masculino. A média de idade foi de 52 anos (28 anos foi a idade mínima e 74 anos, a máxima). Observou-se também que apenas $11 \%(\mathrm{n}=4)$ dos pacientes residem sozinhos. Grande parte é casada ou vive em união estável, o que gera um total de $64 \%$ (n= 23). Com relação ao histórico familiar da psoríase, a maioria, $78 \%(n=28)$, relata não haver casos da doença na família. Exatamente $50 \%(n=18)$ dos entrevistados trabalham, enquanto a outra metade está afastada ou aposentada.

Estudo realizado por Mignorance et al., ${ }^{11}$ ao avaliar a adaptação psicossocial dos pacientes com psoríase, constatou sua relação com a dificuldade nos vínculos psicossociais, inclusive a inserção no mercado de trabalho. Os autores também perceberam que quanto maior a extensão e a gravidade das lesões, maior o prejuízo nas atividades rotineiras, lazer e qualidade geral de vida desses pacientes. Isso poderia explicar o alto percentual de afastamento e aposentadoria (50\%) encontrados no presente estudo.-

Mesmo ciente dos riscos à saúde, $17 \%(\mathrm{n}=6)$ dos pacientes é tabagista e $30 \%(\mathrm{n}=11)$ alega ser ex-tabagista; $66 \%(n=24)$ das pessoas abordadas não consomem nenhum tipo de bebida alcoólica. Dentre os 36 pesquisados, $58 \%(\mathrm{n}=21)$ deles não praticam atividade física e $69 \%$ ( $\mathrm{n}=$ 25) se consideram estressados ou nervosos.

Camerin \& Cestari, ${ }^{12}$ ao avaliarem a psoríase como fator de risco associado ao desenvolvimento de depressão, tabagismo e alcoolismo, observaram dados semelhantes aos encontrados no presente estudo, em que o tabagismo foi relatado por $22 \%$ dos pacientes. Já o histórico de ex-tabagismo foi de $36 \%$, e de depressão, $32 \%$. Estudo realizado por Souza et al. ${ }^{13}$ indicou que os fatores mencionados como desencadeantes ou agravantes das doenças de pele remetem a situações de estresse, estando a maior parte destes relacionados à perda e à separação em todas as áreas de vida - ou seja, o fato de o indivíduo ser estressado ou nervoso, como encontrado no presente estudo, pode ser um gatilho e agravante da psoríase. Não houve associação significativa entre estresse e tabagismo $(\mathrm{p}=0,38)$, ou trabalho $(\mathrm{p}=0,12)$.

A alteração de peso foi encontrada em $67 \%(n=24)$ dos pacientes, dos quais $58 \%(n=14)$ relataram ganho de peso e $42 \%$ ( $n=10)$, perda, não sendo encontrada associação entre esta variável e o estresse $(p=0,63)$. Gisondi et al. ${ }^{14}$ destacam que o aumento de peso em pacientes com psoríase pode participar do desencadeamento da doença, baseado em seu estado pró-inflamatório, ou também poderia ser consequência da psoríase decorrente de desregulações metabólicas, induzidas pelo estado pró-inflamatório, somado ao prejuízo na qualidade de vida e hábitos alimentares do portador desta doença. 
Ao avaliar o estado nutricional, observou-se que $3 \%(n=1)$ dos pacientes estavam com baixo peso, $36 \%(n=13)$ estavam eutróficos, $22 \%(n=8)$ com sobrepeso e $39 \%(n=14)$ com obesidade. Ainda segundo Gisondi et al. ${ }^{14}$, a síndrome metabólica é significativamente mais comum em pacientes psoriásicos, os quais apresentam maior prevalência de hipertrigliceridemia e obesidade abdominal.

Duarte et al. ${ }^{15}$ afirmam que é provável haver relação entre a psoríase e alterações metabólicas e/ou obesidade, com maior morbimortalidade e hospitalização dos pacientes afetados, realçando a necessidade de uma abordagem multidisciplinar na condução do paciente com psoríase, no que concerne, principalmente, às terapêuticas em uso e futuras.

Embora a nutrição seja considerada uma ferramenta no tratamento para a psoríase, não há nenhuma diretriz, nacional ou internacional, que estabeleça uma alimentação adequada a esses pacientes. Alguns autores sugerem que diversos compostos ativos da alimentação brasileira desempenham papéis importantes na fisiopatogenia da psoríase, com a mesma magnitude que o controle do aporte energético da dieta e o consumo de gorduras totais e saturadas contribuem para o controle das DCNTs. ${ }^{4}$

Dentre esses nutrientes, citam-se algumas vitaminas e minerais (vitamina A, E, C e D, e ácido fólico), ácidos graxos poli-insaturados (ômega 3), além de dietas com baixa densidade calórica. Acredita-se que essas vitaminas e os minerais (ferro, cobre, manganês, zinco e selênio) possuem capacidade antioxidante, diminuindo o estresse oxidativo e a produção de espécies reativas de oxigênio, sobretudo na presença de inflamação sistêmica, como é o caso da psoríase..$^{8,16}$

Considerando o fato de a psoríase ser uma doença inflamatória e alguns alimentos apresentarem ação anti ou pró-inflamatória, a alimentação pode ao mesmo tempo prevenir e tratar, como desenvolver e agravar a doença, tornando então a nutrição um fator determinante no tratamento da psoríase. ${ }^{4,5}$

O quadro 1 apresenta os dados relacionados à frequência de consumo de alimentos antiinflamatórios. Os resultados demonstram que o alho $(97 \%$; $n=35)$ e o azeite de oliva $(53 \%$; $n=$ 19) foram os alimentos anti-inflamatórios mais frequentemente consumidos (3 vezes ou mais na semana), enquanto que os demais alimentos tiveram consumo pouco frequente pela maioria da população estudada. 
Quadro 1. Frequência de consumo de alimentos anti-inflamatórios por pacientes acometidos por psoríase. Itajaí-SC, 2014.

\begin{tabular}{|c|c|c|c|c|c|c|}
\hline \multirow[t]{2}{*}{ Alimentos } & \multicolumn{2}{|c|}{$\begin{array}{l}\text { Consome } 3 \times \text { ou } \\
\text { mais na semana }\end{array}$} & \multicolumn{2}{|c|}{$\begin{array}{l}\text { Consome menos } \\
\text { de } 3 \times \text { na semana }\end{array}$} & \multicolumn{2}{|c|}{ Não consome } \\
\hline & $\mathrm{N}$ & $\%$ & $\mathrm{~N}$ & $\%$ & $\mathrm{~N}$ & $\%$ \\
\hline Salmão & 0 & - & 5 & 14 & 31 & 86 \\
\hline Sardinha & 2 & 6 & 15 & 41 & 19 & 53 \\
\hline Atum & 0 & - & 8 & 22 & 28 & 78 \\
\hline Nozes & 10 & 28 & 6 & 17 & 20 & 55 \\
\hline Semente de Chia & 5 & 14 & 4 & 11 & 27 & 75 \\
\hline Chá Verde & 5 & 14 & 4 & 11 & 27 & 75 \\
\hline Azeite de Oliva & 19 & 53 & 9 & 25 & 8 & 22 \\
\hline Óleo de Canola & 4 & 11 & 2 & 6 & 30 & 83 \\
\hline Gengibre & 6 & 17 & 8 & 22 & 22 & 61 \\
\hline Goiaba & 4 & 11 & 11 & 31 & 21 & 58 \\
\hline Alho & 35 & 97 & 0 & - & 1 & 3 \\
\hline Batata Doce & 7 & 19 & 23 & 64 & 6 & 17 \\
\hline Abóbora & 5 & 14 & 21 & 58 & 10 & 28 \\
\hline Cará & 1 & 3 & 5 & 14 & 30 & 83 \\
\hline Pimentão Vermelho & 9 & 25 & 9 & 25 & 18 & 50 \\
\hline Mel & 14 & 39 & 4 & 11 & 18 & 50 \\
\hline Própolis & 3 & 8 & 2 & 6 & 31 & 86 \\
\hline
\end{tabular}

O consumo frequente de alho pelos pacientes da presente pesquisa torna-se um achado importante, pois segundo Lee et al..$^{17}$ (2012), neste alimento já foram encontrados quatro componentes anti-inflamatórios, que atuam especificamente inibindo a produção do oxido nítrico e das prostaglandinas, a expressão do fator de necrose tumoral e das interleucinas, todos grandes participantes da inflamação.

O azeite de oliva extra virgem, segundo alimento mais consumido, possui uma substância chamada oleocanthal, com propriedade anti-inflamatória, além de conter compostos fenólicos ${ }^{18}$ que são potentes antioxidantes e combatem o estresse oxidativo. ${ }^{19}$ 
Dos três peixes analisados no questionário, todos ricos em ômega 3, apenas a sardinha se destacou. Ressalta-se que este nutriente apresenta capacidade anti-inflamatória, tradicionalmente atribuída à inibição da formação de eicosanoides, participantes do processo inflamatório. ${ }^{20}$

O pimentão vermelho, que é rico em carotenoides e tem efeito antioxidante, analgésico e antiinflamatório, ${ }^{21}$ apresentou um consumo frequente por $25 \%$ dos pacientes, que poderia beneficiar muito esses pacientes.

A abóbora, que apresentou consumo esporádico pelos pacientes, também tem grande capacidade anti-inflamatória, antioxidante e antidiabética, pois possui ácido linolênico, oleico, palmítico e flavonoides, ${ }^{22}$ além de ser um alimento comum e de preço acessível.

Outro alimento que teve seu consumo pouco frequente, e é muito difundido atualmente, com grande capacidade anti-inflamatória, antioxidante e até antilipogênica, é a batata doce, cujo efeito antioxidante foi comprovado in vitro e seu poder anti-inflamatório ocorre pela inibição da interleucina- $6 .{ }^{23}$

As nozes foram consumidas frequentemente por $28 \%(\mathrm{n}=10)$ dos pacientes, mas $55 \%(\mathrm{n}=20)$ deles não há consomem, talvez em função do custo. Destaca-se, no entanto, que este alimento possui compostos químicos como fito-esteróis, selênio e tocoferóis, que potencializam sua ação antioxidante inibitória de estresse oxidativo ${ }^{24}$ - inclusive, vem sendo utilizado para atenuar o estresse oxidativo em doenças inflamatórias. ${ }^{25}$

Os outros alimentos não obtiveram um consumo significativo, mas apresentam importantes capacidades anti-inflamatórias que devem ser destacadas, como a Salvia hispanica L., mais conhecida como chia, e o óleo de canola merecem destaque, pois possuem, além de muitas outras propriedades, a característica de conter ômega-3, famoso por sua capacidade anti-inflamatória. ${ }^{26,27}$

O chá verde, se consumido habitualmente, seria de grande valia para esses pacientes, pois tem grandes quantidades de polifenóis e potente capacidade antioxidante e anti-inflamatória. ${ }^{28} \mathrm{~A}$ goiaba, que é rica em polifenóis, com capacidade antioxidante, antimicrobiana e anti-inflamatória, foi consumida por apenas $11 \%$ dos participantes. ${ }^{29}$

Um alimento que pode ser utilizado de várias maneiras, como sucos, salgados e doces, mas foi pouco difundido entre os pacientes, é o gengibre. Segundo estudo realizado na Índia por Jeenak, Liju \& Kuttan, ${ }^{30}$ quando testado em ratos, o gengibre mostrou aumento no sangue da superóxido desmutase, glutationa e glutationa redutase, além de glutationa-S-tranferase, glutationa peroxidase e superóxido desmutase, no fígado, demonstrando significativa redução na inflamação crônica. ${ }^{31}$

O inhame (ou cará) foi um dos menos consumidos pelos pacientes, mas segundo Jin et al., ${ }^{32}$ tem potente ação contra as doenças inflamatórias, em função de sua capacidade de inibir compostos como a ciclioxigenase-1 (COX-1) e a lipoxigenase-5 (LOX-5), dependente da produção de leucotrieno $\mathrm{C}$. 
O mel, o própolis e geleia real já foram amplamente usados na Antiguidade com intuito medicinal. Essa conduta é justificada pelo fato de possuírem propriedades biológicas, como antitumoral, antimicrobiana, anti-inflamatória, efeitos imunomodulatórios entre outros, ${ }^{33}$ mas embora o mel tenha sido consumido frequentemente por cerca de $39 \%$ dos pacientes, o própolis teve seu consumo bastante reduzido.

Quando questionados em relação ao consumo de alimentos inflamatórios três vezes na semana ou mais, o mais citado foi carne de gado, com $58 \%(n=21)$, seguido pelo café $(n=16) 45 \%$, como pode ser observado no quadro 2 , que apresenta dados relacionados ao consumo de alimentos pró-inflamatórios.

Quanto aos alimentos não consumidos nenhuma vez na semana, os mais citados foram: glutamato monossódico, com $97 \%$ (n=35); o chá preto, com $89 \%(n=32)$ e o mate, com $64 \%$ (n=23).

Grande parte dos entrevistados negou consumir alguns dos alimentos inflamatórios, tais como o chá preto e o glutamato monossódico, que tiveram $89 \%(n=32)$ e $97 \%(n=35)$ de rejeição, respectivamente, como pode ser observado no quadro 2.

Quadro 2- Frequência de consumo de alimentos pró-inflamatórios por pacientes acometidos por psoríase. Itajaí-SC, 2014.

\begin{tabular}{|c|c|c|c|c|c|c|}
\hline \multirow{2}{*}{ Alimentos } & \multicolumn{2}{|c|}{$\begin{array}{c}\text { Consome 3 x ou } \\
\text { mais na semana }\end{array}$} & \multicolumn{2}{c|}{$\begin{array}{c}\text { Consome menos } \\
\text { de 3 x na semana }\end{array}$} & \multicolumn{2}{c|}{ Não consome } \\
\hline & $\mathrm{N}$ & $\%$ & $\mathrm{~N}$ & $\%$ & $\mathrm{~N}$ & $\%$ \\
\hline Café preto & 16 & 45 & 3 & 8 & 17 & 47 \\
\hline Chá preto & 2 & 6 & 2 & 6 & 32 & 89 \\
\hline Mate & 9 & 25 & 4 & 11 & 23 & 64 \\
\hline Chocolate & 9 & 25 & 13 & 36 & 14 & 39 \\
\hline Defumados & 7 & 19 & 16 & 45 & 13 & 36 \\
\hline Pimenta & 10 & 28 & 7 & 19 & 19 & 53 \\
\hline Carne de Gado & 21 & 58 & 14 & 39 & 1 & 3 \\
\hline Glutamato monossódico & 1 & 3 & 0 & - & 35 & 97 \\
\hline
\end{tabular}


Corroborando os resultados do presente estudo, Festugato et al. ${ }^{5}$ verificaram que a carne de gado também foi o alimento mais consumido pelos pacientes com psoríase, sendo que o que a diferencia de outras carnes é sua quantidade em ferro, que estaria envolvido na síntese de radicais hidroxila, causando danos às células do intestino. As carnes, quando cozidas ou assadas, perdem água durante o preparo, aumentando o teor de gordura, proteína e o valor calórico. O método de preparo da carne que utiliza altas temperaturas e baixa umidade (fritar, assar, grelhar) contribui para o alto conteúdo dietético de AGEs-Advanced Glycated End-Products, produtos finais da glicação avançada, que danificam as células e se ligam a receptores específicos, causando a produção de citocinas inflamatórias e fatores de crescimento. ${ }^{34-36}$

Ainda são escassos estudos que abordam as implicações dos AGEs em doenças da pele, como a psoríase, mas podemos inferir que as carnes cozidas ou assadas perdem água durante o preparo culinário, aumentando o teor de gordura e proteína, tornando-se fontes de AGEs próinflamatórios. ${ }^{34,37}$

O segundo alimento com maior consumo foi o café. Nele existem grandes quantidade de cafeína, cujo principal mecanismo de ação se deve a sua similaridade estrutural com a molécula de adenosina, podendo ligar-se a seus receptores (A1, A2A), bloqueando-os e, consequentemente, tendo ação estimulante. ${ }^{38}$ A cafeína, que possui efeitos pró-inflamatórios, ao ser administrada em vigência de um processo inflamatório agudo em ratos, aumentou o dano tecidual comprovado pelo aumento dos níveis de RNAm de citocinas TNF-alfa, TNF-beta, linfotoxinabeta, IL-6 e IFNgamma no baço, e aumento de IFNgamma no sangue periférico. ${ }^{39}$ Citocinas, como o IFNgamma, atuam como mitógenos para queratinócitos na psoríase. ${ }^{40}$

Em estudo realizado por Festugato, ${ }^{6}$ alimentos como a pimenta, café e chás (cafeína), glutamato monossódico, carne de gado e alimentos defumados foram suspensos da dieta de pacientes com psoríase, que foram então inseridos em hábitos saudáveis, evidenciando melhora no tratamento. Além disso, observou-se que os pacientes que não apresentavam uma dieta adequada, dada a falta de informação sobre a relevância da nutrição, apresentaram agravamento da psoríase.

Além do consumo dos alimentos citados anteriormente, que podem interferir no tratamento da psoríase, outros fatores podem ajudar na sua evolução, tais como os ambientais, que influenciam no curso e na susceptibilidade do desenvolvimento da doença, incluindo inflamações crônicas, estresse, drogas (betabloqueadores, lítio, interferon e agentes antimalárica), fumo e obesidade. ${ }^{41}$

Tanto a má nutrição, quanto a inflamação são fortemente associadas entre si e podem mudar o compasso nutricional do indivíduo resultando na mesma direção inflamatória. ${ }^{42,43}$ Deste modo, o cuidado nutricional em pacientes com psoríase, juntamente com o controle das variáveis bioquímicas e antropométricas, garante maior estabilidade clínica a esses indivíduos, prevenindo as DCNTs comumente associadas à doença e propiciando maior longevidade com qualidade. ${ }^{44}$ 


\section{Considerações finais}

Dos pacientes estudados, a maioria apresentou estado nutricional comprometido (sobrepeso e obesidade), aumentando os riscos para doenças crônicas relacionadas à obesidade. A má qualidade de vida também pode interferir no agravamento das lesões. Quanto aos alimentos ingeridos, o alto consumo de alho, seguido pelo azeite de oliva, é fator positivo para a melhora da psoríase, mas destaca-se que uma alimentação saudável não está relacionada somente a um tipo de alimento ou nutriente isolado.

Por mais que não tenhamos como quantificar a diminuição das escamas e demonstrar sua correlação com a mudança alimentar, pode-se dizer que a suspensão dos alimentos próinflamatórios, juntamente com a introdução de hábitos nutricionais saudáveis e melhora do estado nutricional, podem contribuir para um melhor resultado no tratamento e consequente elevação da autoestima dos pacientes.

A busca por um tratamento multifatorial é de suma importância, pois os profissionais de várias áreas (médico, psicólogo e nutricionista) podem promover, de forma mais acelerada o bem-estar do paciente.

Destacam-se como limitações do presente trabalho o número reduzido de indivíduos estudados, bem como o referencial teórico escasso sobre o tema. Assim, diante da magnitude do problema, torna-se essencial o desenvolvimento de mais pesquisas que englobem um número maior de participantes e relacionem o estado nutricional de pacientes diagnosticados com psoríase com o consumo de alimentos que possam auxiliar na melhora dessa patologia.

\section{Referências}

1. Miot HA. Genética da psoríase. In: Romiti R, editor. Novos conceitos em psoríase. Rio de Janeiro: Elsevier; 2009. p. 32-38.

2. Silva KS, Silva EAT. Psoríase e sua relação com aspectos psicológicos, stress e eventos da vida. Estud Psicol. 2007; 24(2):257-266.

3. Kremers HM, Mcevoy MT, Dann FJ, Gabriel SE. Heart disease in psoriasis. J. Am. Acad. Dermatol. 2007; 57(2):347-54.

4. Solis MY, Melo NS, Macedo ME, Carneiro FP, Sabbag CY, Lancha-Junior AH, et al. Nutritional status and food intake of patients with systemic psoriasis and psoriatic arthritis associated. Einstein (São Paulo) 2012; 10(1):44-52.

5. Festugato M. Estudo piloto sobre alimentos que devem ser evitados nos portadores de psoríase. An. Bras. Dermatol. 2011; 86(6):1103-1108. 
6. Sterry W, Strober BE, Menter A. Obesity in psoriasis: the metabolic, clinical and therapeutic implications. Report of an interdisciplinary conference and review. International Psoriasis Council. Br. J. Dermatol. 2007; 157:649-5.

7. Shapiro JA, Koepsell TD, Voigt LF. Diet and rheumatoid arthritis in women: a possible protective effect of fish consumption. Epidemiology 1996; 7:256-63.

8. Araujo MLD, Burgos MGPA, Moura ISC. Influências nutricionais na psoríase. An. Bras. Dermatol. 2009; 84(1):90-92.

9. Associação Brasileira de Empresas de Pesquisa [Internet]. Critério de Classificação Econômica: Brasil (Preliminar). [citado em set. 2014]. Disponível em: http://www.abep.org/novo/Content. aspx?ContentID $=886 . \mathrm{m}$

10. Brasil. Ministério da Saúde. Orientações para coleta e análise de dados antropométricos em serviços de saúde: Norma Técnica do Sistema de Vigilância Alimentar e Nutricional - SISVAN. Brasília: Ministério da Saúde; 2011. 71 p.

11. Mingnorance RC, Loureiro SR, Okino L. Pacientes com psoríase: qualidade de vida e adaptação psicossocial. Na. Bras. Dermatol. 2002; 77(2):147-59.

12. Camerin ACS, Cestari TF. A psoríase como fator de risco associado ao desenvolvimento de depressão, tabagismo e alcoolismo. In: XXIII Salão de Iniciação científica UFRGS. [citado em 2011 Out.] Disponível em: http://hdl.handle.net/10183/48875.

13. Souza APFS, Carvalho FT, Rocha KB, Lages MN, Calvetti PU, Castoldi L. Associação de eventos estressores ao surgimento ou agravamento de vitiligo e psoríase. Psico (Porto Alegre) 2005; 36(2):167-174.

14. Gisondi P, Tessari G, Conti A, Piaserico S, Schianchi S, Peserico A. Prevalence of metabolic syndrome in patients with psoriasis: a hospital-based case-control study. Br. J. Dermatol. 2007; 157:68-73.

15. Duarte GV, Follador I, Cavalheiro CMA, Silva TS, Oliveira MFSP. Psoríase e obesidade: revisão de literatura e recomendações no manejo. An. Bras. Dermatol. 2010; 85(3):355-360.

16. Hobold D, Souza MCG. Aspectos nutricionais no tratamento da psoríase. Especialização [monografia]. Florianópolis: UNESC; 2012.

17. Lee Y, Li H, Lim HJ, Lee HJ, Jeon R, Ryu JH. Anti-inflammatory activity of sulfur-containing compounds from garlic. J. Med. Food 2012; 15(11):992-9.

18. Impelizzeri J, Lin JJ. A simple high-performance liquid chromatography method for the determination of throat-burning oleocanthal with probated antiinflammatory activity in extra virgin olive oils. J. Agric. Food Chem. 2006; 54:3204-3208.

19. Bareta P, Septembre-Malaterrea A, Rigouletb M, D'hellencourta L, Priaultb M, Gonthiera M, et al. Dietary polyphenols preconditioning protects 3T3-L1 preadipocytes from mitochondrial alterations induced by oxidative stress. Int. J. Biochem. Cell Bio. 2013; 45(1):167-174.

20. Wall R, Ross RP, Fitzgerald GF, Stanton C. Fatty acids from fish: the anti-inflammatory potential of long-chain omega-3 fatty acids. Nutr. Rev. 2010; 68:280-289. 
21. Hernandez-Ortega M, Ortiz-Moreno A, Hernandez-Navarro MD, Chamorro-Cevallos G, DorantesAlvarez L, Necoechea-Mondragon H. Antioxidant, antinociceptive, and anti-inflammatory effects of carotenoids extracted from dried pepper (Capsicum annuum L.). J. Biomed. Biotechnol. 2012. doi:10.1155/2012/524019.

22. Yadav M, Jain S, Tomar R, Prasad GBKS, Yadav H. Medicinal and biological potential of pumpkin: an updated review. Nutr. Res. Rev. 2010; 23:184-190.

23. Ju J, Yoon H, Park H, Kim M, Shin H, Park K, et al. Anti-Obesity and Antioxidative Effects of Purple Sweet Potato Extract in 3T3-L1 Adipocytes In Vitro. J. Med. Food. 2011; 14:1097-1106.

24. Freitas JB, Naves MMV. Composição química de nozes e sementes comestíveis e sua relação com a nutrição e saúde. Rev. Nutr. 2010; 23(2):269-279.

25. Soory M. Nutritional antioxidants and their applications in cardiometabolic diseases. Infect Disord. Drug Targets 2012; 12(5):388-401.

26. Mohd AN, Yeap SK, Ho WY, Beh BK, Tan SW, Tan SG. The promising future of Chia, Salvia hispanica L. J. Biomed. Biotechnol. 2012. doi: 10,1155 / 2012/171956

27. Yoona J, Leeb EJ, Kimc HD, Leed JH, Kangc H. Polyunsaturated fatty acid-enriched diet therapy for a child with epilepsy. Brain Dev. 2014; 36(2):163-6.

28. Bornhoeft J, Castaneda D, Nemoseck T, Wang P, Henning SM, Hong MY. The protective effects of green tea polyphenols: lipid profile, inflammation, and antioxidant capacity in rats fed an atherogenic diet and dextran sodium sulfate. J. Med. Food 2012; 15(8):726-732.

29. Flores G, Dastmalchi K, Wu SB, Whalen K, Dabo AJ, Reynertson KA, et al. Phenolic-rich extract from the Costa Rican guava (Psidium friedrichsthalianum) pulp with antioxidant and anti-inflammatory activity. Potential for COPD therapy. Food Chem. 2013; 141(2):889-95.

30. Jeena K, Liju VB, Kuttan R. Antioxidant, anti-inflammatory and antinociceptive activities of essential oil from ginger. Indian J. Physiol. Pharmacol. 2013; 57(1):51-62.

31. Mccook-Russel KP, Nair MG, Facey PC, Bowen-Forbes CS. Nutritional and nutraceutical comparison of Jamaican Psidium cattleianum (strawberry guava) and Psidium guajava (common guava) fruits. Food Chem. 2012; 134(2):1069-73.

32. Jin M, Lu Y, Yang JH, Jo TH, Park YI, Lee CK, et al. Anti-inflammatory activity of 6-hydroxy-2,7dimethoxy-1,4-henanthraquinone from tuberous roots of yam (Dioscorea batatas) through inhibition of prostaglandin D and leukotriene C production in mouse bone marrow-derived mast cells. Arch. Pharm. Res. 2011; 34(9):1495-501.

33. Viuda-Martos M, Ruiz-Navaras Y, Fernandez-Lopes J, Perez-Alvez JA. Functional properties of honey, propolis, and royal jelly. J. Food Sci. 2008; 73(9):R117-24.

34. Vlassara H, Cai W, Crandall J, Goldberg T, Oberstein R, Dardaine V. Inflamatory mediators are induced by dietary glycotoxins, a major risk factor for diabetic angiopathy. Proc. Natl. Acad. Sci. USA 2002; 99:15596-601. 
35. Barbosa JHP, Oliveira SL, Seara LT. O papel dos produtos finais da glicação avançada (AGEs) no desencadeamento das complicações vasculares do diabetes. Arq. Bras. Endocrinol. Metab. 2008; 52:940-50.

36. Instituto Nacional do Câncer. Falando sobre o câncer do intestino: orientações úteis ao usuário fatores de risco e proteção. Rio de Janeiro: INCA; 2013. 35 p.

37. Roça RO. Composição química da carne [Internet]. In: Universidade Estadual Paulista "Júlio de Mesquita Filho" - UNESP. Departamento de Gestão e Tecnologia Agroindustrial. Artigos técnicos, científicos e teses. [citado em out. 2014] Disponível em: http://www.fca.unesp.br/Home/Instituicao/ Departamentos/Gestaoetecnologia/Teses/Roca102.pdf.

38. Alves RC, Casa S, Oliveira B. Benefícios do café na saúde: mito ou realidade? Quím. Nova 2009; 32:2169-80.

39. Ohta A, Lukashev D, Jackson EK, Fredholm BB, Sitkovsky M. 1,3,7- Trimethylxantine (caffeine) may exacerbate acute inflammatory liver injury by weakening the physiological immunosupressive mechanism. J. Immunol. 2007; 179:7431-8.

40. Sociedade Brasileira de Dermatologia. Consenso brasileiro de psoríase e guias de tratamento. Rio de Janeiro: Sociedade Brasileira de Dermatologia; 2006.

41. Chandranand V, Raychaudihuri SP. Geoepidemiology and environmental factors of psoriasis and psoriatic arthritis. J. Autoimmun 2010; 34(3):314-21.

42. Ling PR, Smith RJ, Kie S, Boyce P, Bistrian BR. Effects of protein malnutrition on IL-6-mediated signaling in the liver and the systemic acute-phase response in rats. Am. J. Physiol. Regul. Integr. Comp. Physiol. 2004; 287(4):R801-8.

43. Kalantar-Zadeh K, Cano NJ, Budde K, Chazot C, Kovesdy CP, Mak RH, Mehrotra R, Raj DS, Sehgal AR, Stenvinkel P, Ikizler TA. Diets and enteral supplements for improving outcomes in chronic kidney disease. Nat. Rev. Nephrol. 2011; 37(3):369-84.

44. Solis MY, Sabbag CY, Feangella VS. Evidence of the impact of nutrition in psoriasis. Rasbran. 2013; 5(1):41-51.

Recebido: 28/8/2015

Revisado: 30/9/2015

Aprovado: 12/10/2015 